\title{
Ovarialkarzinom: Hormonersatz verlängert das Leben
}

\author{
Hormonersatztherapie (HRT): Ja oder nein? Bei Frauen mit Ovarialkarzinom \\ bestand über Nutzen und Risiko dieser Behandlung bisher keine Klarheit. Die \\ AHT-Studie liefert neue Erkenntnisse.
}

$B^{\mathrm{e}}$ ei prämenopausalen Frauen kann die operative Entfernung der Eierstöcke Wechseljahrsbeschwerden hervorrufen. Bisher war man unsicher, ob eine HRT zur Behandlung dieser Beschwerden das Rezidivrisiko steigert, zumal einige Ovarialkarzinomzellen Hormonrezep-

toren aufweisen. In der AHT(„adjuvant hormone therapy")-Studie wurden die Effekte der HRT auf die Prognose von Frauen mit epithelialem Ovarialkarzinom untersucht. 150 prä- und postmenopausale Frauen, bei denen ein epitheliales Ovarialkarzinom vor höchstens 9

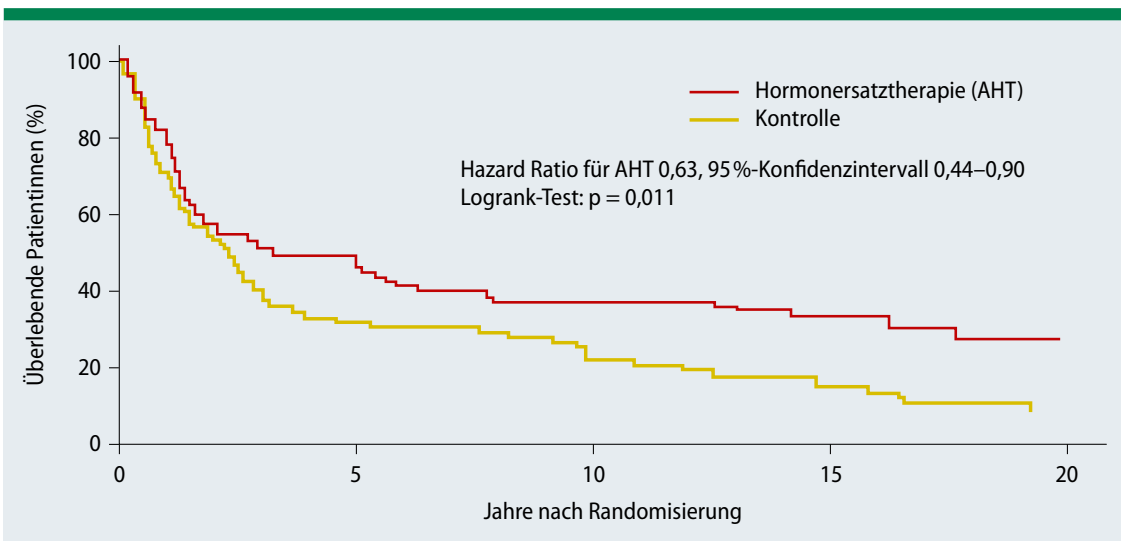

Abb. 1: Patientinnen mit Hormonersatztherapie lebten signifikant länger.

\section{Langzeitergebnisse zur Stellung der minimal- invasiven Hysterektomie bei Uteruskarzinomen}

\begin{abstract}
Der Stellenwert der minimal-invasiven im Vergleich zur offenen Hysterektomie wurde auf Basis des US-amerikanischen SEER-Registers untersucht.
\end{abstract}

$T^{\mathrm{r}}$ rotz potenzieller Vorteile der laparoskopischen Hysterektomie bei Korpuskarzinomen gibt es bisher keine Daten zur Sicherheit in nicht selektierten Kollektiven. Die SEER-Medicare-Datenbank ermöglicht es, die Häufigkeit der verschiedenen Prozeduren, die assoziierte Morbidität und das Langzeitüberleben zu ermitteln. 6.304 Frauen $\geq 65$ Jahre mit der Diagnose eines Gebärmutterkarzinoms (Stadium I-III, etwa $90 \%$ endometrioid) hatten sich zwischen 2006 und 2011 einer Hysterektomie unterzogen. Verglichen wurden perioperative Morbizeitüberleben nach offener $(\mathrm{n}=4.139$, 65,7\%) und laparoskopischer Operation $(\mathrm{n}=2.165,34,3 \%)$. Der Anteil minimalinvasiver Eingriffe stieg kontinuierlich von 9,3\% im Jahr 2006 auf $61,7 \%$ im Jahr 2011. Der Anteil roboterassistierter Eingriffe lag in den letzten 3 Jahren über $60 \%$. Im Vergleich zur offenen ging die minimal-invasive Hysterektomie mit einer niedrigeren Komplikationsrate (22,7 vs. $39,7 \%$; $<<0,001)$ und einer geringeren perioperativen Mortalität (0,6 vs. $1,1 \%)$ einher. Die Patientinnen wurden dität, adjuvante Therapien und Lang-
Monaten diagnostiziert worden war, erhielten randomisiert entweder eine HRT mit konjugiertem Östrogen und Norgestrel, einem Östradiol-Pflaster oder einem Östradiol-Implantat über 5 Jahre oder keine adjuvante Therapie. $77 \%$ der Patientinnen waren postmenopausal. Das mediane Alter lag bei 58,7 Jahren.

Bei Patientinnen mit HRT war das Gesamtüberleben im Vergleich zur Kontrollgruppe signifikant länger (Hazard Ratio [HR] 0,63; $\mathrm{p}=0,011$; nach Adjustierung für prognostisch bedeutsame Faktoren: HR 0,45; $p<0,001)$. Der Unterschied zwischen den Gruppen nahm mit der Zeit zu (Abb. 1). Ähnliches war beim rezidivfreien Überleben zu beobachten. Die Rate unerwünschter Ereignisse war niedrig und in den Gruppen vergleichbar.

Fazit: Eine HRT ist bei Frauen mit schweren menopausalen Symptomen nach Therapie ihres Ovarialkarzinoms sicher und könnte sogar das Gesamtüberleben verlängern. Judith Neumaier

Eeles RA et al. Adjuvant Hormone Therapy May Improve Survival in Epithelial Ovarian Cancer: Results of the AHT Randomized Trial. J Clin Oncol. 2016;33(35):4138-44.

nach minimal-invasiver Operation jedoch häufiger adjuvant bestrahlt (34,3 vs. $31,3 \%)$ oder erhielten eine Brachytherapie (33,6 vs. $31,0 \%$; $p<0,05)$. Die roboterassistierte Operation war mit höheren Komplikationsraten verbunden (23,7 vs. $19,5 \% ; p=0,03)$. Insgesamt gab es aber keine Assoziation zwischen minimal-invasivem Vorgehen und Gesamt- oder krebsspezifischer Mortalität.

Fazit: Die minimal-invasive Hysterektomie ist heute in den USA das bevorzugte Verfahren zur Operation von Uteruskarzinomen. Sie ist diesen Daten zufolge im Vergleich zur offenen Hysterektomie nicht mit einem schlechteren Langzeitüberleben verbunden. Friederike Klein

Wright JD et al. Comparative Effectiveness of Minimally Invasive Hysterectomy for Endometrial Cancer. J Clin Oncol. 2016;34(10):1087-96. 